\title{
Is the time an impact factor for improvement mandibular denture retention?
}

\begin{abstract}
Complete denture is still the most common way to treat edentulism. Therefore, achieving optimum retention and stability is an essential and dynamic issue to improve the quality of life.

The aim of this study was to evaluate denture retention for one year follow up.

Material and methods: 12 patients from the outpatient clinic of Prosthodontics Department (Faculty of Dentistry, Minia University, Egypt) were included in this study. They were selected out of sixty examined patients. The age ranges were 55-67 years. They have completely edentulous ridges and having Class III broad and rounded ridge with adequate height and width according to Cawood and Howell classification of edentulous mandibles. The protocol of the study was approved by the Local Research and Ethics Committee. Patients received complete maxillary and mandibular dentures following the conventional steps of complete denture construction. A force measurement gauge was used for measuring the retention for mandibular denture. The retention was recorded after two weeks from denture insertion, three, six and twelve months. Data was statistically analyzed using t test at a significance level $<0.05$.
\end{abstract}

Results: The retention values for the mandibular dentures showed a statistically insignificant change with time along the follow period $(\mathrm{p}=0.065)$.

Conclusion: The present results showed that the time cannot be considered as an effective factor for improvement of mandibular denture retention.
Volume 9 Issue 3 - 2018

\author{
Amal M Elsawy, ${ }^{1,2}$ Noura A Omar,' Iman A El \\ asfahani $^{2}$ \\ 'Department of Prosthodontics, College of Dentistry, Princess \\ Nourah Bint Abdul Rahman University, Saudi Arabia \\ ${ }^{2}$ Department of Prosthodontics, Minia University, Egypt
}

\begin{abstract}
Correspondence: Amal M Elsawy, Department of Prosthodontics, College of Dentistry, Princess Nourah Bint Abdul Rahman University, Saudi Arabia, Tel 54I786473, Email amalsawy@pnu.edu.sa
\end{abstract}

Received: June 01,2018 | Published: June 26, 2018

\section{Introduction}

Complete denture is still the most common way to treat edentulism. Therefore, achieving optimum retention and stability of full dentures is an essential and dynamic issue to improve the quality of life for completely edentulous patient. Many physical forces and factors have been credited causing or enhancing complete denture retention, e.g. atmospheric pressure, adhesion, cohesion, wettability, gravity, surface tension, viscosity, base adaptation, border seal and muscular control. Most of them depend on factors controlling the flow of the interposed fluid. The better the fit to the tissue, and the better the linear extent of the seal at the border, the better the denture will resist short term displacing forces. ${ }^{1}$ Many researches focused on the importance of time in improvement of mandibular denture retention. They agreed that, flow is time dependent phenomenon and it is dynamic issue..$^{2-4}$ The duration of force application affects the amount of separation of dentures from mucosa. For example; before detachment has occurred, a reseating force like chewing is applied, the displacement will only be transitory and the point of collapse might not be reached. Consequently, care is needed in judging retention. ${ }^{5}$ Evaluation of patients' acceptance and satisfaction with their complete denture therapy is limited by the various methods used in collecting and rating all the influential factors, ${ }^{6}$ such as number of corrections after insertion, psychological characteristics of patients, self-evaluation of affective state or of quality of life, demographic and socioeconomic factors (e.g., age, gender, level of education, level of income, transcultural differences), patient expectation of dentures, quality of denture construction, occlusal factors, factors that are connected with anatomic and physiologic characteristics of the patient (e.g., degree of alveolar ridge resorption, quality of saliva, tongue hypertrophy, status of oral mucosa, quality of denture-bearing area). ${ }^{7,8}$ Previous studies showed that patients with complete dentures were less satisfied; there was a weak negative correlation between the mandibular denture retention and quality of life..$^{9-13}$ Perea et al (2013) investigated the differences in impact on oral health-related quality of life among complete denture wearers depending on their socio-demographic characteristics, prosthetic-related factors and oral status. The results revealed that the prosthesis' location significantly influenced the overall patient satisfaction, the lower dentures being the less comfortable. The authors concluded that the use of conventional complete dentures brings negative impacts in the quality of life of elderly patients, mainly in case of lower prostheses due to weak retention..$^{14}$ The idea of the present research is to answer the question; is the time an impact factor for improvement mandibular denture retention? The aim of this study was to evaluate denture retention for one year follow up.

\section{Material and methods}

The sample of this study included twelve patients from the outpatient clinic of prosthodontics department. They were selected out of thirty examined patients. The age range was 55-67 years (4males and 8 females). They have completely edentulous ridges and having Class III broad and rounded ridge with adequate height and width according to Cawood and Howell classification of edentulous mandibles..$^{15}$ All selected patients were new denture wearers. Patients with good general health were included in the study. They were free from systemic diseases, which were confirmed by preoperative 
complete urine and blood analysis, as well as kidney and liver function tests. Controlled diabetic patients were included in this study. The mucosa of the edentulous ridge of each patient was clinically examined to detect any signs of inflammation, ulceration, flabbiness of the tissues or any sharp bony or tender areas. The study protocol was approved by the Local Research and Ethics Committee, and all patients signed written, informed consent.

\section{Complete denture construction}

Patients received complete maxillary and mandibular dentures following the conventional steps of complete denture construction. ${ }^{14}$ Function technique was followed for making preliminary and secondary impressions. ${ }^{16}$ Maxillomandibular relations (orientation, vertical and horizontal relations) were obtained. The record bases and the occlusion rims were transferred to a semi-adjustable articulator (Whip Mix 8500, Whip Mix Corporation, Louisville, KY USA) by using a quick mount face bow(1 8645 Quick Mount Face-Bow, Whip Mix Corporation, Louisville, KY USA ) and check bite centric relation record. The artificial cross linked acrylic teeth with cusp angle $20^{\circ}$ were used and arranged. Trial dentures were evaluated intraorally for phonetics, aesthetics, occlusal vertical dimension and centric relation. Protrusive and lateral records were made to set the articulator's condylar elements and to achieve a balanced occlusal arrangement After the wax up, the dentures were processed, finished and polished. At the denture placement appointment, clinical remounting was done using a new centric relation record and final occlusal adjustments and refinements were done on the articulator to provide balanced occlusion. Finished dentures were delivered to the patients and any necessary adjustments were carried out. Patients were recalled for any complaints, which were resolved accordingly during the first week. After two weeks from denture insertion, retention measurements were recorded. The patients were scheduled for follow-up visits every 3 months during the first year.

\section{Denture retention measurement}

The lower denture was poured into stone to form a cast after blocking the undercuts with molding wax. On the cast, the relative geometric center of the lower denture was determined by connecting the anterior and posterior extremes of the denture (midline and retromolar pads respectively) to form a triangle. The geometric center of the triangle was then identified on the cast by the intersection of all straight lines that divide it into two parts of equal moment about the line, which corresponds to the geometric center of the denture. Three small wrought wire eyelets were incorporated into the denture as follows; one at the midline lingual to central incisors and the other two distal to the second molars corresponding to the right and left retromolar pad areas. Wrought wires ( $1 \mathrm{~mm}$ diameter) were attached to the eyelets, such that they intersect and bend to form a C-shaped loop at a point $2 \mathrm{~cm}$ above the occlusal plane vertical to and in line with the predetermined geometric center. Measurements of retention were made using a force measurement gauge (Digital Force Gauge Device Model 47544, Extech Instrument Cooperation, Taiwan) Figure 1. The patient was seated in an upright position with the head resting firmly against the head rest. The mandibular denture was positioned correctly on the tissues and the patient was asked to rest his tongue passively in the floor of the mouth with its tip adjacent to the anterior denture teeth. A hook adapter was attached to the universal sensing pull head at the end of the force gauge. The hook was used to engage the denture via the wrought wire loop without altering denture position or retention. It was then pulled in a vertical direction until the denture was dislodged from its seating position. This force was measured in Newton $(\mathrm{N})$ and recorded as the lower denture's retention. Ten records from 2 interexaminer prosthodontists were taken and a mean was calculated. The patients were instructed to relax for one minute between each record. The mean denture retention of the 10 dentures was calculated.

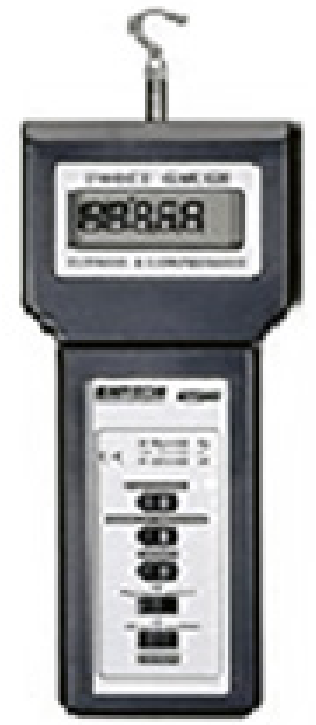

Figure I Digital force gauge devise model 47544.

\section{Statistical analysis}

The data were collected, tabulated and statistically analyzed. T-test was carried out to study the significant difference during the followup period for retention. P-Value $<0.05$ was considered as statistically significant (Figure 2).

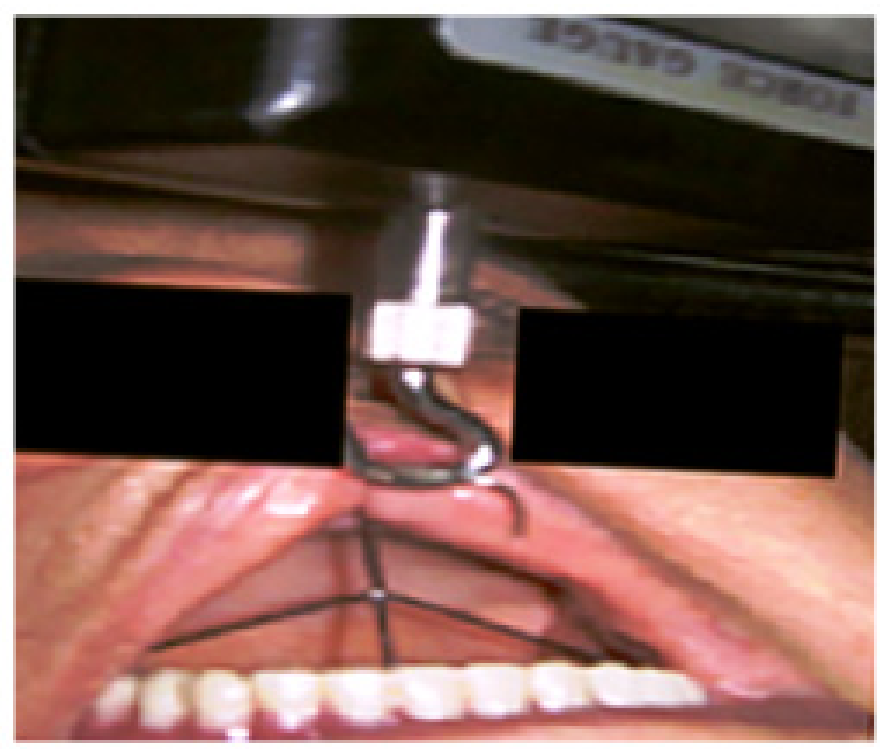

Figure 2 Measuring retention of mandibular denture. 


\section{Results}

Descriptive statistics were presented in the form of mean and standard deviations. The retention was recorded after two weeks from denture insertion then after three, six and twelve months. The results of retention recorded for the conventional complete dentures along the follow up period are shown in Table 1. The retention values for the dentures were $3.297 \pm 0.515,3.633 \pm 0.549,3.987 \pm 0.562$ and $4.107 \pm 0.507 \mathrm{~N}$ for 2 weeks, 3 months, 6months and 12 months respectively. The effect of time on the retention values for the conventional complete dentures showed a statistically insignificant change with time along the follow up period $(\mathrm{p}=0.065$ for the conventional denture respectively) (Figure 3).

Table I Mean and standard deviation (SD) of retention recorded for the conventional dentures along the follow up period

\begin{tabular}{|c|c|c|c|c|c|c|c|c|c|}
\hline \multirow{2}{*}{$\begin{array}{l}\text { Follow up time period } \\
\text { Statistical values }\end{array}$} & \multicolumn{2}{|c|}{2 weeks } & \multicolumn{2}{|c|}{3 months } & \multicolumn{2}{|c|}{6 months } & \multicolumn{2}{|c|}{12 months } & \multirow{2}{*}{$\mathbf{P}$ value } \\
\hline & Mean & SD & Mean & $\mathrm{SD}$ & Mean & SD & Mean & SD & \\
\hline Retention measurements & 3.297 & 0.515 & 3.633 & 0.549 & 3.987 & 0.562 & 4.107 & 0.507 & 0.065 \\
\hline
\end{tabular}

*: significant at $\mathrm{P}<0.05 ; \mathrm{P}>0.05$ (non-significant)

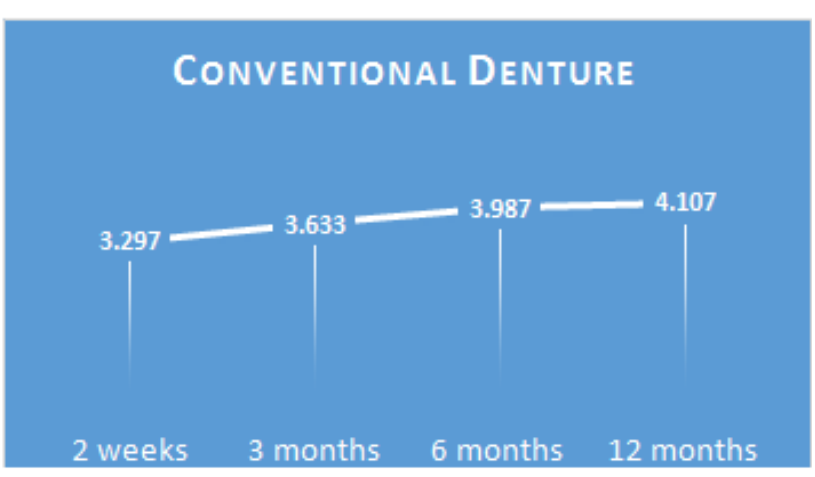

Figure 3 A line charts showing mean retention values recorded for the conventional dentures along the follow up period.

\section{Discussion}

For many decades, complete dentures have always had many problems. They induce unfavorable forces resulting in loss of masticatory performances and influence psychological state of patient's and quality of life. Adaptation to the conventional removable complete dentures is a complex learning process. There was a positive correlation between a denture's retention and quality of life. ${ }^{17}$ On the other hand, patients who have originally adapted to wearing complete dentures may become maladaptive with time, due to the continual residual ridge resorption, intra oral physiological changes and the development of an altered muscle pattern. ${ }^{18}$ The interference factors that may affect the retention and stability of the mandibular denture were excluded in the selected patients. According to Batt et al., ${ }^{19}$ successful treatment with conventional complete dentures depends on both denture quality and oral conditions. Complete denture retention is the resistance to displacement of the denture base away from the ridge which is the main source of psychological comfort for the patient. ${ }^{20}$ This was shown to have a significant influence on usage of and satisfaction with new dentures..$^{21,22}$ There was conflict regarding the useful measures for determining the patient satisfaction with new denture. In the present article, the retention force was used as an objective value to evaluate changes in denture adaptation. The results of the present study revealed that time may be not an effective factor for improvement of denture retention. ${ }^{11}$ However, it must be emphasized that successful prosthodontics rehabilitation for edentulism is multifactorial, and the clinical characteristics of alveolar ridges or denture adaptation may not predict treatment outcomes. ${ }^{20}$

\section{Conclusion}

The present results emphasized that the time cannot be an effective factor for improvement of mandibular denture retention. The complete denture patient couldn't be informed that time will improve their dentures retention.

\section{Acknowledgements}

None.

\section{Conflict of interest}

The author declares that there is no conflict of interest.

\section{References}

1. Lindstrom RE, Pawelchak J, Heyd A, et al. Physical-Chemical aspects of denture retention and stability: a review of the literature. J Prosthet Dent. 1979;42(4):371-375.

2. Rendell JK, Gay T, Grasso JE, et al. The effect of denture adhesive: on mandibular movement during chewing. J Am Dent Assoc. 2000;131:981-986.

3. Özcan M, Kulak Y, De Baat $\mathrm{C}$, et al. The effect of a new denture adhesive on bite force until denture dislodgement. J Prosthodont. 2005;14(2):122-126.

4. Nicolas E, Veyrune JL, Lassauzay C. A Six-Month Assessment of Oral Health-Related Quality of Life of Complete Denture Wearers Using Denture Adhesive: A Pilot Study. J Prosthodont. 2010;19(16):443-448.

5. Darvell BW, Clark RK. The physical mechanisms of complete denture retention. British Dental Journal. 2000;189(5):248-252.

6. Yoshida M, Sato Y, Akagawa Y, et al. Correlation between quality of life and denture satisfaction in elderly complete denture wearers. Int $J$ Prosthodont. 2001;14:77-80.

7. Sato Y, Hamada S, Akagawa $Y$, et al. A method for quantifying overall satisfaction of complete denture patients. J Oral Rehabil. 2000;27(11):952957.

8. van der Waas MAJ. The influence of psychologic factors on patient satisfaction with complete dentures. J Prosthet Dent. 1990;63(5):545548.

9. Berg E. The influence of some anamnestic, demographic, and clinical variables on patient acceptance of new complete dentures. Acta Odontol Scand. 1984;42(2):119-127. 
10. Pietrokovski J, Harfin J, Mostavoy R, et al. Oral findings in elderly nursing home residents in selected countries: quality of and satisfaction with complete dentures. J Prosthet Dent. 1995;73(2):132-135.

11. de Souza e Silva ME, de Magalhaes CS, Ferreira e Ferreira E. Complete removable prostheses: from expectation to (dis)satisfaction. Gerodontology. 2009;26(2):143-149.

12. Al Baker A. The oral health-related quality of life in edentulous patients treated with Conventional complete dentures. Gerodontology. 2013;30:61-66

13. Celebic A, Knezovic-Zlataric D, Papic M, et al. Factors Related to Patien Satisfaction with Complete Denture Therapy. J Gerontol A Biol Sci Med Sci. 2003;58(10):948-953.

14. Perea C, Suárez-García MJ, Del Río J, et al. Oral health-related quality of life in complete denture wearers depending on their socio-demographic background, prosthetic-related factors and clinical condition. Med Oral Patol Oral Cir Bucal. 2013;18(3):371-80.

15. Cawood JJ, Howell RA. A classification of the edentulous jaws. Int J Oral Maxillofac Surg. 1988;17(4):232-236.

16. Zarb GA, Bolender CL, Eckert SK. Prosthodontic treatment for edentulous patients complete denture and implant supported prosthses. $12^{\text {th }}$ ed Newdelhi: Elsevier; 2004.
17. Wolff A, Gadre A, Begleiter A, et al. Correlation between patient satisfaction with complete dentures and denture quality, oral condition, and flow rate of submandibular/sublingual salivary glands. Int $J$ Prosthodont. 2003;16(1):45-48.

18. Wyatt CC. The effect of prosthodontic treatment on alveolar bone loss: a review of the literature. J Prosthet Dent.1998;80(3):362-366.

19. de Baat C, van Aken AA, Mulder J, et al. Prosthetic condition" and patient's judgment of complete dentures. J Prosthet Dent. 1997;78(5):472-478.

20. Mathew L, Unni KN, Vijayakumar. A mathematical derivation to prove reduced denture retention in $\mathrm{v}$ shaped palate. J Indian Prosthodont Soc. 2014;14(2):169-71

21. Fenlon MR, Sherriff M, Walter JD. An investigation of factors influencing patients' use of new complete dentures using structural equation modeling techniques. Community Dent Oral Epidemiol. 2000;28(2):133-140.

22. Fenlon MR, Sherriff M. An investigation of factors influencing patients' satisfaction with new complete dentures using structural equation modelling. J Dent. 2008;36(6):427-434. 